\title{
Temporal Networks: Slowing Down Diffusion by Long Lasting Interactions
}

\author{
Naoki Masuda, ${ }^{1}$ Konstantin Klemm, ${ }^{2}$ and Víctor M. Eguíluz ${ }^{3}$ \\ ${ }^{1}$ Department of Mathematical Informatics, The University of Tokyo, 7-3-1 Hongo, Bunkyo, Tokyo 113-8656, Japan \\ ${ }^{2}$ Bioinformatics, Institute of Computer Science, Leipzig University, Härtelstraße 16-18, 04107 Leipzig, Germany \\ ${ }^{3}$ Instituto de Física Interdisciplinar y Sistemas Complejos IFISC (CSIC-UIB), E07122 Palma de Mallorca, Spain
}

(Received 13 May 2013; published 29 October 2013)

\begin{abstract}
Interactions among units in complex systems occur in a specific sequential order, thus affecting the flow of information, the propagation of diseases, and general dynamical processes. We investigate the Laplacian spectrum of temporal networks and compare it with that of the corresponding aggregate network. First, we show that the spectrum of the ensemble average of a temporal network has identical eigenmodes but smaller eigenvalues than the aggregate networks. In large networks without edge condensation, the expected temporal dynamics is a time-rescaled version of the aggregate dynamics. Even for single sequential realizations, diffusive dynamics is slower in temporal networks. These discrepancies are due to the noncommutability of interactions. We illustrate our analytical findings using a simple temporal motif, larger network models, and real temporal networks.
\end{abstract}

DOI: 10.1103/PhysRevLett.111.188701

PACS numbers: 89.75.Hc, 05.45.-a, 02.10.Ud

Interactions in social, biological, and engineered networks are often being established and dismantled in a temporal sequence rather than being static properties. Such systems represented as networks with a sequence of time stamped interacting node pairs are called temporal networks [1]. The specifics of temporal interactions affect accessibility [2] and dynamical processes on networks such as epidemic spreading [1,3-5], synchronization [6], random walks [7], and consensus [8-12]. In the context of numerical simulations of population dynamics, including networked dynamical systems (e.g., coupled oscillators), the comparison of aggregate and temporal dynamics is tantamount to the choice of synchronous or asynchronous numerical schemes for updating states of the agents. Although the effects of the two numerical schemes have been examined, the analytical results are scarce [13-15], and the evidence mostly remains numerical [16-19].

Here, we are interested in generic effects to which such temporal interactions may give rise. A comparison is made with the corresponding aggregate dynamics where all interactions are present permanently. We consider dynamical systems with diffusive couplings and theoretically analyze their spectral properties, which represent various dynamics such as synchronization, random walks, and diffusive processes $[20,21]$. We show that diffusive dynamics is slower for the temporal network (i.e., asynchronous update) than for the aggregate network (i.e., synchronous update) and find qualitatively different effects, even after averaging over random temporal sequences of purely linear interactions.

Published by the American Physical Society under the terms of the Creative Commons Attribution 3.0 License. Further distribution of this work must maintain attribution to the author $(s)$ and the published article's title, journal citation, and DOI.
General framework.-We consider linear dynamics under a set $\mathcal{M}$ of interaction matrices. For a time interval of length $\tau$, a matrix $M^{(0)}$ drawn from $\mathcal{M}$ determines the dynamics. Then, another matrix $M^{(1)}$ is drawn from $\mathcal{M}$ and is active for time $\tau$, and so forth. The $N$-dimensional state vector $\boldsymbol{x}(t)$ evolves according to

$$
\dot{\boldsymbol{x}}(t)=M^{(\mid t / \tau])} \boldsymbol{x}(t),
$$

where $t \geq 0$. Rounding to the next lowest integer is denoted by $\lfloor\cdot]$, so $\lfloor t / \tau\rfloor$ is the number of time intervals of length $\tau$ before time $t$. The parameter $\tau$ measures the ratio of time scales of the dynamics of $x$ and of the evolution of the interactions.

Each specific real-world scenario of duration $r \tau$ produces a particular sequence $S=\left(M^{(0)}, M^{(1)}, \ldots, M^{(r-1)}\right)$ of interaction matrices, where $r \in \mathbb{N}$. The dynamics given by Eq. (1) has the formal solution $\boldsymbol{x}(r \tau)=T(S ; \tau) \boldsymbol{x}(0)$ with the matrix

$$
T(S ; \tau)=\exp \left(\tau M^{(r-1)}\right) \cdots \exp \left(\tau M^{(0)}\right)
$$

being the time evolution operator for a given sequence $S$. An initial condition $\boldsymbol{x}(0)$ maps to the same $\boldsymbol{x}(r \tau)$ at time $r \tau$ under the dynamics with constant matrix $(r \tau)^{-1} \ln T(S ; \tau)$, which we call the effective matrix of sequence $S$.

Random sequences with replacement.-Being interested in the generic effects of temporal networks, we first consider ensembles of sequences with uniform probability, that is, sequences generated by drawing uniformly and independently with replacement from $\mathcal{M}$. Starting from an initial condition $\boldsymbol{x}(0)$, the expected state at time $\tau$ is given by

$$
\langle\boldsymbol{x}(\tau)\rangle=\hat{T}(\tau) \boldsymbol{x}(0)
$$

with the time evolution operator now averaged over all interactions 


$$
\hat{T}(\tau)=|\mathcal{M}|^{-1} \sum_{M \in \mathcal{M}} \exp (\tau M) .
$$

The effective matrix for the temporal dynamics is

$$
\hat{M} \equiv \tau^{-1} \ln \hat{T}(\tau) .
$$

This is to be compared to the dynamics $\dot{\boldsymbol{x}}=M^{*} \boldsymbol{x}$ under the aggregate matrix

$$
M^{*} \equiv|\mathcal{M}|^{-1} \sum_{M \in \mathcal{M}} M,
$$

where all interactions are permanently present. The time evolution operator is given by

$$
T^{*}(\tau)=\exp \left(\tau M^{*}\right) .
$$

Whenever $M^{*} \neq \hat{M}$, aggregate dynamics and temporal dynamics are different already at the level of the expectation over random sequences of interactions. Formally, such a difference is rooted in the fact that averaging over interactions and integration of the dynamics do not commute in general.

Edge sequences with replacement.- - Let us consider an undirected multigraph $G=(V, E)$ with nodes $V=$ $\{1, \ldots, N\}$ and a multiset $E$ of edges given as unordered pairs of nodes. We are considering a multiset so that the same unordered pair can appear as several edges. A single diffusive coupling between $i$ and $j$, induced by a network edge $e=\{i, j\}$, is represented by a matrix $M^{(e)}$ with $M_{i i}^{(e)}=$ $M_{j j}^{(e)}=-1, M_{i j}^{(e)}=M_{j i}^{(e)}=+1$, and zero at all other entries. Using a homogeneous coupling strength $\sigma$, i.e., replacing each $M^{(e)}$ by $\sigma M^{(e)}$, amounts to a general time rescaling $t \rightarrow t / \sigma$. Also replacing $\tau$ by $\tau / \sigma$, all resulting eigenvalues are simply scaled up by $\sigma$. The Laplacian of the aggregate network is given by $L=-\sum_{e \in E} M^{(e)}$. Taking into account that squaring the matrix yields $M^{(e)} M^{(e)}=-2 M^{(e)}$ [22], the temporal evolution operator under coupling $e$ and time $\tau$ is given by

$$
\exp \left(\tau M^{(e)}\right)=I+\alpha(\tau) M^{(e)}
$$

where

$$
\alpha(\tau) \equiv \frac{1-\exp (-2 \tau)}{2} .
$$

By combining Eqs. (4) and (8), we obtain the ensemble averaged temporal evolution operator for a single application of the edge as follows:

$$
\hat{T}(\tau)=|E|^{-1} \sum_{e \in E} \exp \left(\tau M^{(e)}\right)=I+\alpha(\tau) M^{*},
$$

with the aggregate matrix $M^{*}=|E|^{-1} \sum_{e \in E} M^{(e)}=$ $-|E|^{-1} L$. The effective interaction matrix is obtained as

$$
\hat{M}=\tau^{-1} \ln \hat{T}(\tau)=\tau^{-1} \ln \left[I+\alpha(\tau) M^{*}\right] .
$$

Remarkably, $\hat{M}$ is obtained from the aggregate matrix $M^{*}$ purely by functional calculus. Therefore, the aggregate and temporal matrices have identical eigenspaces. Each eigenvalue $\mu^{*}$ of the aggregate matrix $M^{*}$ maps to an eigenvalue $\hat{\mu}$ of the temporal matrix $\hat{M}$ according to

$$
\hat{\mu}=f\left(\mu^{*}, \tau\right) \equiv \tau^{-1} \ln \left[1+\alpha(\tau) \mu^{*}\right] .
$$

Before analyzing Eq. (12) further, we remark that $-2 \leq$ $\mu^{*} \leq 0$. An eigenvalue -2 is obtained if and only if $|E|=1$, i.e., for a single interaction. For arbitrary $\tau \neq 0$, the function $f$ has fixed points $\mu=0$ and $\mu=-2$. For $-2<\mu^{*}<0, f\left(\mu^{*}, \tau\right)$ monotonically increases with $\tau$, and $f\left(\mu^{*}, \tau\right) \rightarrow 0$ as $\tau \rightarrow \infty$.

In the limiting case $\tau \rightarrow 0$, temporal and aggregate dynamics coincide as $f\left(\mu^{*}, \tau\right) \rightarrow \mu^{*}$. An increase of $\tau$ has the following consequences. First, the dynamics slows down because eigenvalues move closer to 0 . Second, dynamical behavior qualitatively changes because the slowing down of modes is not linear; i.e., the ratios between eigenvalues change. The fastest modes are least affected when $\tau$ is increased. However, the nonlinearity is significant only for eigenvalues of $M^{*}$ that are close to -2 .

If $\left|\mu^{*}\right|$ is sufficiently small, we can apply the first order approximation $\ln (1+x) \approx x$ to Eq. (12) to obtain

$$
\hat{\mu} \approx \rho(\tau) \mu^{*}
$$

where $\rho(\tau)=(2 \tau)^{-1}[1-\exp (-2 \tau)]$. To the extent that the approximation is valid for all eigenvalues of $M^{*}$, the temporal matrix itself approximates to $\hat{M} \approx \rho(\tau) M^{*}$.

Let us comment on the relevance of the approximation for large networks. The eigenvalues of $M^{*}$ are lower bounded [23] by $-\max \left\{d_{i}+d_{j}:\{i, j\} \in E\right\} /|E|$, where $d_{i}$ is the degree of node $i$, i.e., the number of edges incident on $i$. For eigenvalue $\mu^{*}$, this bound translates into $\left|\mu^{*}\right| \leq$ $2 d_{\max } /|E|$, where $d_{\max }$ is the maximum degree in the network. The approximation given by Eq. (13) improves for a growing network size if $d_{\max } /|E| \rightarrow 0$; i.e., the fraction of edges owned by each node tends to zero. Then, all eigenvalues of $M^{*}$ tend to zero, and the expected temporal dynamics is just a time-rescaled version of the aggregate dynamics.

Otherwise, a node accumulates a finite fraction of the edges with growing $N$, a phenomenon called Bose-Einstein condensation in networks [24]. Then, an eigenvalue $\mu^{*}$ of $M^{*}$ may remain finitely separated from zero even when $N \rightarrow \infty$.

The largest deviation from the approximation is obtained when the fastest eigenvalue of the aggregate matrix $M^{*}$ remains at -1 [25] while the slowest eigenvalue tends to zero when $N \rightarrow \infty$. In this case, the slow and fast modes are decelerated by the factors $\rho(\tau)$ and $f(-1, \tau)$, respectively, when passing from the aggregate to temporal dynamics. The ratio between the deceleration factors is maximum in the limit of large $\tau$, where we obtain 


$$
\lim _{\tau \rightarrow \infty} \frac{\rho(\tau)}{f(-1, \tau)}=\frac{-1}{2 \ln (1 / 2)} \approx 0.72
$$

The slow modes are decelerated less than fast modes, and the ratio between the eigenvalues corresponding to the slow and fast modes does not fall below $72 \%$ of the original value. The bound is attained in the case of a star, which has the Laplacian eigenvalues 0,1 , and $N$, translating into eigenvalues $0, \mu_{\text {slow }}^{*}=-1 /(N-1)$, and $\mu_{\text {fast }}^{*}=$ $-N /(N-1)$ for matrix $M^{*}$. Networks possessing a large ratio $\mu_{\text {fast }}^{*} / \mu_{\text {slow }}^{*}$, including scale-free networks, are known to be difficult to synchronize in coupled chaotic dynamics $[20,26]$. Although $\mu_{\text {fast }}^{*}$ does not remain $O(1)$ as $N \rightarrow \infty$, such networks deviate from Eq. (13) more than homogeneous networks of the same size do.

Small example.-For illustration, we consider a network with $N=3$ nodes and two edges $E=\{\{1,2\},\{2,3\}\}$. The eigenvalues of the aggregate matrix are equal to $\mu^{*}=0$, $-1 / 2$, and $-3 / 2$. For the temporal dynamics averaged over random sequences with replacement, Eq. (12) results in $\hat{\mu}=0, f(-1 / 2, \tau)$, and $f(-3 / 2, \tau)$. As the theory predicts, $\hat{\mu}$ (dotted curves in Fig. 1) is closer to zero than $\mu^{*}$ is, and $\hat{\mu}$ approaches zero as $\tau$ increases. In particular, the spectral gap, i.e., the eigenvalue with the smallest nonzero absolute value, is smaller for the temporal than for the aggregate dynamics; the spectral gap gives the time scale of relaxation.

If we select $S=\left(M^{\{1,2\}}, M^{\{1,2\}}\right)$ or $S=\left(M^{\{2,3\}}, M^{\{2,3\}}\right)$ in the sampling with replacement, the spectral gap trivially vanishes. For sequences $S=\left(M^{\{1,2\}}, M^{\{2,3\}}\right)$ and $S=$ $\left(M^{\{2,3\}}, M^{\{1,2\}}\right)$, the eigenvalues of the effective matrix are plotted by the solid curves in Fig. 1. Also, in this sampling scheme, in which we avoid multiple sampling of the same edge and the resulting loss of connectivity of the entire

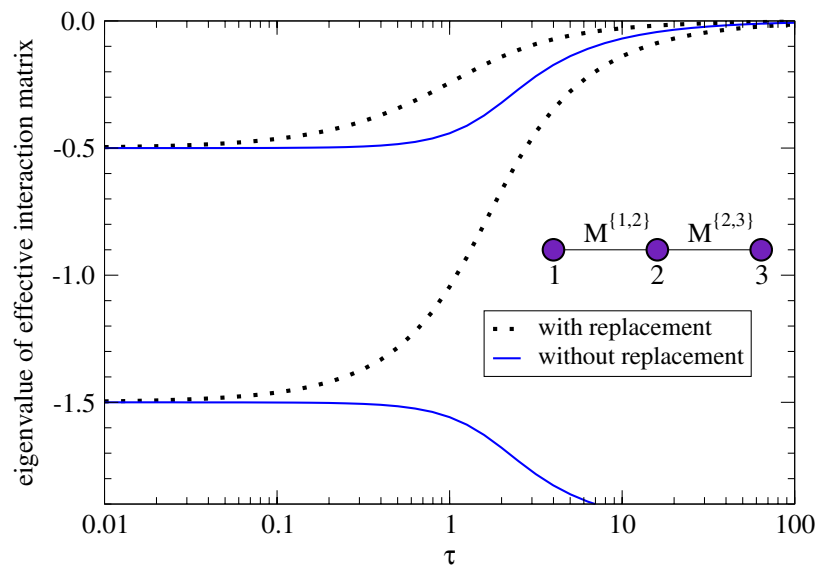

FIG. 1 (color online). Dependence of the spectrum of effective interaction matrices on the temporal parameter $\tau$. We show the two nonzero eigenvalues of effective interaction matrices for a network with two edges connecting three nodes. Dotted curves: Random sequences with replacement. Solid curves: Random sequences without replacement. network, the spectral gap, at least, is smaller for the temporal than for the aggregate dynamics.

Edge sequences without replacement.-We now consider edge sequences sampled without replacement. In the previous example, it corresponds to the two sequences containing both edges (solid curves in Fig. 1). For a general network and an arbitrary permutation $S=$ $\left(M^{(0)}, M^{(1)}, \ldots, M^{(|E|-1)}\right)$ of its edges, the spectral gap of the effective temporal matrix in this case is smaller than or equal to that of the aggregate matrix.

To show this, we use Eqs. (8) and (9) to write the time evolution operator according to Eq. (2) as

$$
T(S ; \tau)=\left[I+\alpha(\tau) M^{(|E|-1)}\right] \cdots\left[I+\alpha(\tau) M^{(0)}\right] .
$$

If $\phi$ is a so-called spectral function, $\phi\left(e^{A} e^{B}\right) \geq\left|\phi\left(e^{A+B}\right)\right|$ holds true for general matrices $A$ and $B$ [27,28]. The product of the two largest eigenvalues is a spectral function. Because the largest eigenvalue of the evolution operator for the Laplacian dynamics is equal to unity for both aggregate and temporal dynamics, with the right eigenvector being $(1 \cdots 1)^{\top}$, the second largest eigenvalue of $e^{M^{(i)}} e^{M^{(j)}}$ is at least that of $e^{M^{(i)}+M^{(j)}}$ for arbitrary $i$ and $j$. Therefore, in terms of the absolute value, the spectral gap for the effective matrix $(|E| \tau)^{-1} \ln T(S ; \tau)$ is at most $\left|\mu^{*}\right|$. This result implies that the dynamics on an arbitrary temporal network is slower than (or at least not faster than) that on the aggregate network.

When $\tau \rightarrow 0$, we can approximate the right-hand side of Eq. (15) using $\alpha(\tau) \approx \tau$ by

$$
I+\alpha(\tau) \sum_{i=1}^{|E|} M^{(i)}=I+\tau|E| M^{*} \approx T^{*}(|E| \tau) .
$$

Therefore, the temporal dynamics approaches the aggregate dynamics as $\tau \rightarrow 0$ as in the case of the random sampling with replacement.

Application to model and real networks.-For two model temporal networks and two real temporal networks, the (negative) spectral gaps $\mu^{*}$ and $\hat{\mu}$ are compared in Fig. 2. For random sampling without replacement, we appropriately normalized the spectral gap of $\tau^{-1} \ln T(S ; \tau)$ by dividing by $|E|$.

The first model network is generated on a realization of the Erdős-Rényi random graph having 500 nodes and 1978 edges, which is the aggregate network. Because adjacent nodes usually have multiple edges in real data [1], we assumed 10 edges between each pair of adjacent nodes in the aggregate random graph. Figure 2(a) indicates that, under the sampling both with and without replacement, $|\hat{\mu}|$ i.e., the spectral gap for the temporal network averaged over 1000 sequences (thick and thin dashed lines), is consistently smaller than $\left|\mu^{*}\right|$ (thick solid line) for any $\tau$. The results are qualitatively the same for a scale-free temporal network [Fig. 2(b)] constructed by placing 10 edges on each edge of an aggregated network, which is a realization of the Barabási-Albert model [29] having 500 nodes and 


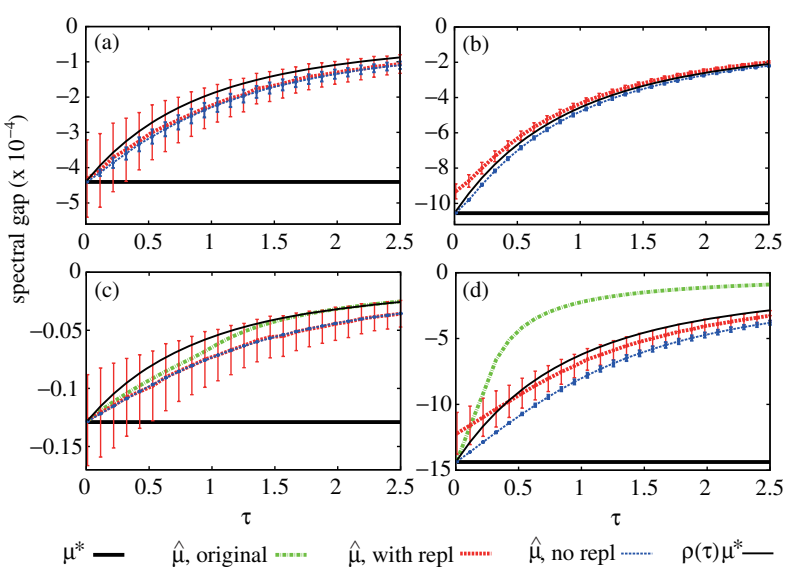

FIG. 2 (color online). The (negative) spectral gap of the aggregate and temporal Laplacian dynamics for four networks. The error bars indicate the standard deviation calculated on the basis of $10^{3}$ realizations of edge sequences. (a) Results for a random temporal graph having $N=500$ nodes and $|E|=19780$ edges. (b) Results for a scale-free temporal network with $N=500$ and $|E|=19990$. We set the parameters of the Barabási-Albert model to $m=m_{0}=4$. The degree $d$ obeys a power law with exponent -3 [29]. (c) Results for human interaction data with $N=104$ and $|E|=782682$ among students, staff, and faculty members at the Massachusetts Institute of Technology. The data were produced by the Reality Mining Project [30]. Although the original network contains 106 subjects, we used the largest connected component containing 104 subjects. (d) Results for the human interaction data during a conference recorded in the SocioPatterns Project [31]. Although the original data have 113 nodes and 20818 edges, we excluded one node and the link emanating from this node, possessing two edges on it before the analysis. This is because the spectral gap value is very sensitive to such a nearly isolated node. The modified network has $N=112$ and $|E|=20816$.

1990 edges. The results for human interaction data obtained from the Reality Mining Project, having 104 nodes and 782682 edges [30], and those from the SocioPatterns Project, having 112 nodes and 20816 edges [31], are shown in Figs. 2(c) and 2(d), respectively.

First of all, $\mu^{*}$ is independent of $\tau$ by definition (thick solid lines). For all the networks, we verified that $|\hat{\mu}|<$ $\left|\mu^{*}\right|$ holds true for the temporal networks derived from the real-world interaction sequences [dash-dotted lines labeled "original" in Figs. 2(c) and 2(d)] and individual temporal networks generated by the sampling without replacement (ensemble averaged values are shown by the thin dashed lines). It should be noted that it is not the case for the sampling with replacement (averages are shown by the thick dashed lines). Second, for both the sampling with replacement and without replacement, Fig. 2 indicates that $|\hat{\mu}|<\left|\mu^{*}\right|$ holds true on the average and that $|\hat{\mu}|$ decays toward zero as $\tau$ increases. Third, the original sequence of edges and random sequences both with and without replacement yield values of $\hat{\mu}$ approaching $\mu^{*}$ as $\tau \rightarrow 0$. All these numerical results are consistent with our theoretical results. Finally, Eq. (13) suggests that $\hat{\mu}$ in the case of sampling with replacement is approximated by $\rho(\tau) \mu^{*}$. Figure 2 shows that this is a reasonable approximation (thin solid lines).

The error bars in Fig. 2 are large in some cases, indicating that the $\hat{\mu}$ value depends much on the individual sequence. This is also implied by the discrepancy between the $\hat{\mu}$ values for the real temporal networks [dash-dotted lines in Figs. 2(c) and 2(d)] and those averaged over samples (thick and thin dashed lines). When any pair of $M^{(i)}(1 \leq i \leq|E|)$ commutes, the time evolution operators for the aggregate and temporal dynamics [Eqs. (7) and (15)] are identical. This is tantamount to saying that the pairwise noncommutability of graphlets may be a main source of the discrepancy between $\hat{\mu}$ and $\mu^{*}$ and also that between $\hat{\mu}$ for different temporal networks sharing the same aggregate network.

Discussion.-We have quantitatively shown that the effect of temporal networks on diffusive dynamics is considerable. Our results imply that synchronization is more difficult in temporal than in the corresponding aggregate networks for general $\tau>0$, in agreement with the numerical results in Ref. [6]. The slowing down due to temporal dynamics is also observed in other diffusive dynamics such as random walks [7], the voter model [8,9], and the naming game [11]. Our theoretical results also enlarge previous theoretical understanding of synchronization dynamics in temporal networks. The theoretical results obtained under the framework of switching topology mostly treat the case of fast switching, which corresponds to $\tau \rightarrow 0$ in the present study [32-35]. In the limit $\tau \rightarrow 0$, the stability of the aggregate Laplacian matrix is theoretically sufficient for synchronization in temporal networks [32-35], which is consistent with our results. We obtained a quantitative theory for general $\tau$ without strong constraints like simultaneous diagonalizability of the graphlets [36]. Going beyond purely diffusive dynamics, the spectral framework is relevant for systems with local dynamics at each node. A stability criterion for the synchrony of such dynamics across nodes is based on the eigenvalues of the coupling matrix [37]. Tests with simple systems of chaotic oscillators provide the first evidence that the present spectral analysis for time-dependent coupling matrices correctly combines with that approach (see the Supplemental Material [38]). Finally, real interaction sequences show correlated patterns [4,39]. To reveal the relationship between such correlated patterns and the behavior of individual temporal sequences is warranted for future work.

We thank the SocioPatterns collaboration (http:// www.sociopatterns.org) for providing the data set. We also acknowledge financial support provided by Grants-in-Aid for Scientific Research (No. 23681033) from MEXT, Japan, the Nakajima Foundation, VolkswagenStiftung, and MINECO (Spain) and FEDER (EU) through the MODASS Project (No. FIS2011-24785). 
[1] P. Holme and J. Saramäki, Phys. Rep. 519, 97 (2012).

[2] H. H. K. Lentz, T. Selhorst, and I. M. Sokolov, Phys. Rev. Lett. 110, 118701 (2013).

[3] M. Karsai, M. Kivelä, R. K. Pan, K. Kaski, J. Kertész, A.-L. Barabási, and J. Saramäki, Phys. Rev. E 83, 025102 (2011).

[4] G. Miritello, E. Moro, and R. Lara, Phys. Rev. E 83, 045102(R) (2011).

[5] N. Masuda and P. Holme, F1000 Prime Rep. 5, 6 (2013).

[6] N. Fujiwara, J. Kurths, and A. Díaz-Guilera, Phys. Rev. E 83, 025101 (2011)

[7] M. Starnini, A. Baronchelli, A. Barrat, and R. PastorSatorras, Phys. Rev. E 85, 056115 (2012).

[8] J. Fernández-Gracia, V. M. Eguíluz, and M. S. Miguel, Phys. Rev. E 84, 015103 (2011).

[9] T. Takaguchi and N. Masuda, Phys. Rev. E 84, 036115 (2011).

[10] G. J. Baxter, J. Stat. Mech. 09 (2011) P09005.

[11] A. Baronchelli and A. Díaz-Guilera, Phys. Rev. E 85, 016113 (2012).

[12] S. K. Maity, T. V. Manoj, and A. Mukherjee, Phys. Rev. E 86, 036110 (2012).

[13] C. L. Nehaniv, Int. J. Algebra Comput. 14, 719 (2004).

[14] A. A. Hansson, H. S. Mortveit, and C. M. Reidys, Adv. Compl. Syst. 8, 521 (2005).

[15] M. Cao, A. S. Morse, and B. D. O. Anderson, IEEE Trans. Autom. Control 53, 1826 (2008).

[16] B. A. Huberman and N. S. Glance, Proc. Natl. Acad. Sci. U.S.A. 90, 7716 (1993).

[17] M. A. Nowak, S. Bonhoeffer, and R. M. May, Proc. Natl. Acad. Sci. U.S.A. 91, 4877 (1994).

[18] H. Bersini and V. Detours, Artif. Life IV, 382 (1994).

[19] D. Cornforth, D. G. Green, and D. Newth, Physica (Amsterdam) 204D, 70 (2005).

[20] L. Donetti, F. Neri, and M. A. Muñoz, J. Stat. Mech. 08 (2006) P08007.

[21] J. A. Almendral and A. Díaz-Guilera, New J. Phys. 9, 187 (2007).
[22] For directed networks, the single-edge Laplacian satisfies $M^{(i)} M^{(i)}=-M^{(i)}$; the factor 2 disappears as compared to the case of the undirected network.

[23] W. N. Anderson, Jr. and T. D. Morley, Linear Multilinear Algebra 18, 141 (1985).

[24] G. Bianconi and A.-L. Barabási, Phys. Rev. Lett. 86, 5632 (2001).

[25] The lower bound [23] of the eigenvalues of $M^{*}$ implies $\mu^{*} \geq-\max \left\{d_{i}+d_{j}:\{i, j\} \in E\right\} /|E| \geq-1-|E|^{-1}$.

[26] T. Nishikawa, A.E. Motter, Y.-C. Lai, and F.C. Hoppensteadt, Phys. Rev. Lett. 91, 014101 (2003).

[27] J. E. Cohen, S. Friedland, T. Kato, and F. P. Kelly, Linear Algebra Appl. 45, 55 (1982).

[28] N. Bebiano, J. da Providência, Jr., and R. Lemos, Linear Algebra Appl. 376, 265 (2004).

[29] A.-L. Barabási and R. Albert, Science 286, 509 (1999).

[30] N. Eagle and A. Pentland, Pers. Ubiquit. Comput. 10, 255 (2006).

[31] L. Isella, J. Stehlé, A. Barrat, C. Cattuto, J.-F. Pinton, and W. Van den Broeck, J. Theor. Biol. 271, 166 (2011)

[32] I. V. Belykh, V.N. Belykh, and M. Hasler, Physica (Amsterdam) 195D, 188 (2004).

[33] D. J. Stilwell, E. M. Bollt, and D. G. Roberson, SIAM J. Appl. Dyn. Syst. 5, 140 (2006).

[34] P. So, B.C. Cotton, and E. Barreto, Chaos 18, 037114 (2008).

[35] M. Frasca, A. Buscarino, A. Rizzo, L. Fortuna, and S. Boccaletti, Phys. Rev. Lett. 100, 044102 (2008).

[36] J. Zhao, D. J. Hill, and T. Liu, Automatica 45, 2502 (2009).

[37] L. M. Pecora and T.L. Carroll, Phys. Rev. Lett. 80, 2109 (1998).

[38] See Supplemental Material at http://link.aps.org/ supplemental/10.1103/PhysRevLett.111.188701 for additional details on nonlinear local dynamics.

[39] J.-P. Eckmann, E. Moses, and D. Sergi, Proc. Natl. Acad. Sci. U.S.A. 101, 14333 (2004). 